\title{
The Role of Perioperative Transesophageal Echocardiography in Pediatric Cardiac Surgery: Should It be an Essential Monitor in Pediatric Cardiac Surgeries?
}

Indications for transesophageal echocardiography (TEE) in patients with congenital heart disease (CHD) include the following: ${ }^{1}$

- Diagnostic indications

- Nondiagnostic transthoracic echocardiography in suspected CHD

- Evaluation of intracardiac or extracardiac baffles following the Fontan, Senning or Mustard procedure

- Other general indications, not characteristic only of CHD (infective endocarditis, prosthetic valve function, prior cardioversion, etc.)

- Perioperative indications

- Transesophageal echocardiography-guided interventions (e.g. atrial septal defect closure)

According to 'Practice Guidelines for Perioperative TEE', most cardiac defects requiring repair under cardiopulmonary bypass are a category 1 indication for intraoperative TEE including precardiopulmonary bypass and postcardiopulmonary bypass imaging. Category 1 is defined as that being supported by the strongest evidence or expert opinion substantiating that TEE is useful in improving clinical outcomes. ${ }^{2}$ According to 'American College of Cardiology (ACC)/American Heart Association (AHA) guidelines for the clinical application of echocardiography', monitoring and guidance during cardiothoracic procedures associated with the potential for residual shunts, valvular regurgitation, obstruction, or myocardial dysfunction, are a class I indication for TEE in pediatric patients with CHD. Class I indication is defined as conditions for which there is evidence and/or general agreement that a given procedure or treatment is useful and effective. ${ }^{3}$

Transesophageal echocardiography is an essential monitoring and diagnostic device in pediatric cardiac surgery for confirming preoperative diagnoses, demonstrating previously unappreciated anatomic details, formulating surgical plans, evaluating immediate operative results, detecting significant residual lesions, and guiding surgical revisions. The anesthesiologist echocardiographer should have a thorough knowledge of the spectrum of congenital heart defects and a rich experience in two-dimensional, pulsed wave, continuous wave and color Doppler echocardiography of $\mathrm{CHD}^{4}$

The intraoperative TEE performed just before surgical intervention may confirm or exclude preoperative transthoracic echocardiography (TTE) findings and assess associated anomalies, size of heart chambers, the immediate preoperative hemodynamics, direction of shunts and ventricular function of the patient. The findings can be directly demonstrated to the surgeon for immediate review just prior to commencement of the operation. ${ }^{4}$ Because of the excellent resolution of TEE, new abnormalities are sometimes diagnosed prerepair that call for a revision of the surgical plan. Preoperative TEE may also facilitate the placement of central venous catheters, selection of anesthetic agents, and use of preoperative inotropic support by demonstrating ventricular systolic function and size. ${ }^{4}$ It can help to guide the hemodynamic management before cardiopulmonary bypass. Transesophageal echocardiography is a sensitive method for detecting the presence of intracardiac air and aiding its removal before removal of extracorporeal circulation.

Performance of TEE in the patient with CHD immediately after surgery, but before chest closure is a great contributor to the outcome for congenital heart surgery. Post-procedure TEE is used to evaluate the repair and to guide post-bypass hemodynamic management. The echocardiographer must have an excellent understanding of the surgical repair to interpret intraoperative findings. ${ }^{4}$ Based upon the TEE and clinical findings, the surgeon, in conjunction with the TEE echocardiographer anesthesiologist, determines whether the surgical repair is acceptable. By identifying the site, severity and mechanism of residual problems, TEE offers substantial utility in detection of residual problems in need of reoperation. It provides the opportunity to detect significant and potentially treatable disease before disconnection of bypass cannulae, sternal closure and return to the intensive care unit. ${ }^{5}$ In addition, it assesses cardiac function and may aid in the diagnosis of cardiac rhythm abnormalities. Obtaining real-time information 
allows the surgeon to correct inadequate repairs and prevent or treat surgical complications before the patient leaves the operating room, therefore, reducing the need of reoperations. Stevenson et $\mathrm{al}^{6}$ investigated 230 patients who underwent surgical correction of congenital cardiopathies and observed that at the end of the procedure, TEE detected residual cardiac defects which determined new interventions for correction, in $7 \%$ of the patients. Transesophageal echocardiography assists in evaluating the intracardiac results of minimally invasive cardiac surgical procedures, in which there is limited direct visualization of the heart.

Transesophageal echocardiography is useful for intraoperative management of Fontan procedure (for single ventricle situations), especially for evaluation of the right atrium pulmonary artery anastomosis, monitoring of hemodynamics and wall motion after cardiopulmonary bypass, evaluation of pulmonary artery flow velocity patterns, for determination of the required shape and size of the interatrial shunt (fenestration), and evaluation of interatrial fenestration flow. ${ }^{7}$

Intraoperative TEE enables measurement of homograft size. ${ }^{8}$ The homograft is stored in a freezer and thawed before being used. Transesophageal echocardiography is used to measure the left or right ventricular outflow tract diameter for sizing the homograft before the patient is placed on cardiopulmonary bypass. This allows a homograft of correct size to be selected at the beginning of the operation and thawed.

When postoperative TTE is not feasible, TEE may be used to monitor hemodynamic changes as inotropic drugs or ventilator settings are adjusted. In the critically ill postoperative patient with limited transthoracic views, TEE permits assessment of ventricular function and assists in determining appropriate timing and hemodynamic effect of sternal closure (delayed sternal closure) or discontinuation of ventricular assist device or extracorporeal membrane oxygenation. Transesophageal echocardiography may assist in the further characterization of unexpected/unusual findings or pathology following congenital cardiac surgery which are identified or suspected, but not adequately defined by transthoracic imaging. These possible postoperative clinical situations include the following: ${ }^{9}$

- Residual atrial shunting, especially in patients with unexplained cyanosis

- Possible pulmonary venous obstruction

- Residual ventricular septal defects

- Atrioventricular and semilunar valve abnormalities

- Mechanism of left or right ventricular outflow tract obstruction
- Prosthetic valve malfunction

- Possible infective endocarditis

The patient with intracardiac or extracardiac baffles or conduits (Fontan, Senning, Mustard or Rastelli operation) may have pathways that are particularly difficult to image with TTE. In patients after Fontan operation, TEE is very useful in assessing baffle leak or dehiscence, thrombus or obstruction. Patients with atrial baffles are often at risk for atrial tachycardia and intracardiac thrombi. Transesophageal echocardiography is the method of choice to detect intracardiac thrombi after Fontan operation, because it provides an excellent view of the atrial structures and central venous connections. ${ }^{10}$ Transesophageal echocardiography is helpful in excluding the presence of thrombus prior to cardioversion in these patients.

Although the procedure of TEE probe insertion is generally safe, there are a few inherent risks to placement of the probe, including pharyngeal and/or laryngeal trauma, dental injuries, esophageal trauma, bleeding, arrhythmias, respiratory distress and hemodynamic effects.

Technological advancements have allowed better TEE imaging small probe sizes, and multiplane capability. These have significantly improved patient safety and success of cardiac surgery in infants and children by providing substantial information in the perioperative period. Transesophageal echocardiography has become a critical diagnostic and perioperative management tool for patients with congenital heart disease undergoing cardiac and noncardiac surgical procedures. In noncardiac surgery, TEE can be used to evaluate ventricular volume, function, underlying pathology, and to assess any residual lesions. ${ }^{11}$ Perioperative TEE is one of the greatest advances in modern cardiac anesthesia and it is now the standard of care for intraoperative assessment of patients with CHD.

\section{REFERENCES}

1. Flachskampf FA, Wouters PF, Edvardsen T, Evangelista A, Habib G, Hoffman P, Hoffman R, Lancellotti P, Pepi $\mathrm{M}$; European Association of Cardiovascular Imaging Document Reviewers: Erwan Donal and FAUSTO Rigo. Recommendations for transesophageal echocardiography: EACVI update 2014. Eur Heart J Cardiovas Imag 2014 Apr;15(4):353-365.

2. Thys D, Abel M, Bollen B, et al. Practice guidelines for perioperative transesophageal echocardiography. Anesthesiology 1996;84:986-1006.

3. Cheitlin M, Alpert J, Armstrong W, et al. ACC/AHA guidelines for the clinical application of echocardiography: a report of the American College of Cardiology/American Heart Association Task Force on Practice Guidelines 
(committee on clinical application of echocardiography). Circulation 1997;95:1686-1744.

4. Joffe D. Intraoperative transesophageal echocardiography. In: Lewin MB, Stout K. Echocardiography in Congenital Heart Disease. Elsevier Saunders; 2012. p. 31-57.

5. Ayres NA, Miller-Hance W, Fyfe DA, Stevenson JG, Sahn DJ, Young LT, Minich LL, Kimball TR, Geva T, Smith FC, et al. Indications and guidelines for performance of transesophageal echocardiography in the patient with pediatric acquired or congenital heart disease. J Am Soc Echocardiogr 2005 Jan;18(1):91-98.

6. Stevenson JG, Sorensen GK, Gartman DM, Hall DG, Rittenhouse EA. Transesophageal echocardiography during repair of congenital cardiac defects: identification of residual problems necessitating reoperation. J Am Soc Echocardiogr 1993 Jul-Aug;6(4):356-365.

7. Kawahito S, Kitahata H, Tanaka K, Nozaki J, Iseki A, Oshita S. Transesophageal echocardiography for intraoperative management of the fenestrated Fontan procedure. J
Cardiothoracic and Vascular Anesthesia 2000 Aug;14(4): 457-459.

8. Click RL, Oh JK. Intraoperative echocardiography (Chapter 21). In: Oh JK, Seward JB, Tajik AJ, editors. The Echo Manual. 3rd ed. Lippincott WIlliams \& Wilkins; 2006. p. 368-382.

9. Miller-Hance WC, Russel IA. Intraoperative and postoperative transesophageal echocardiography in congenital heart disease. In: Wong PC, Miller-Hance WC, editors. Transesophageal Echocardiography for Congenital Heart Disease: Springer; 2014. p. 383-395.

10. Balling G, Vogt M, Kaemmerer H, Eicken A, Meisner H, Hess J. Intracardiac thrombus formation after the Fontan operation. J Thorac Cardiovasc Surg 2000 Apr;119(4 pt 1): 745-752.

11. Russell I, Foster E, Rouine-Rapp K. Assessment of congenital heart disease in the adult patient. In: Savage RM, Aronsen S, Shermane SK, editors. Comprehensive Textbook of Perioperative Transesophageal Echocardiography. 2nd ed. Lippincott Williams and Wilkins; 2011. p. 390-405.

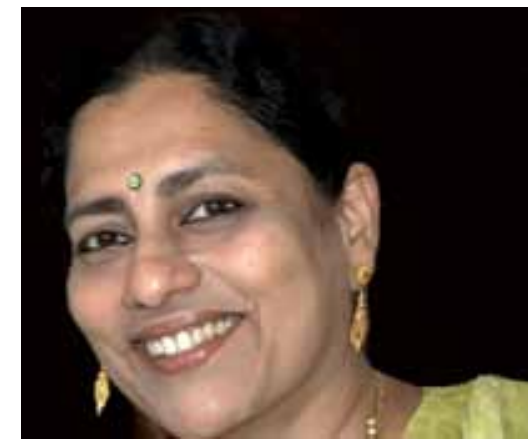

Rupa Sreedhar

Professor and Head

Department of Anesthesia

Sree Chitra Tirunal Institute for Medical Sciences and Technology Thiruvananthapuram, Kerala, India 